\title{
Swift monitoring of Supergiant Fast X-ray Transients: the out-of-outburst behaviour and the flares from IGR J17544-2916 and XTE J1739-302
}

\author{
L. Sidoli ${ }^{*},{ }^{a}$ P. Romano, ${ }^{b}$ G. Cusumano, ${ }^{b}$ V. Mangano, ${ }^{b}$ S. Vercellone, ${ }^{a}$ A. Paizis, ${ }^{a}$ A. \\ Pellizzoni, ${ }^{c}$ J.A. Kennea, ${ }^{d}$ D.N. Burrows, ${ }^{d}$ H.A. Krimm, ${ }^{e f g}$ N. Gehrels,${ }^{g}$ C. Guidorzi, ${ }^{h}$ \\ P.A. Evans ${ }^{i}$ \\ ${ }^{a}$ INAF, Istituto di Astrofisica Spaziale e Fisica Cosmica, \\ Via E. Bassini 15, I-20133 Milano, Italy \\ ${ }^{b}$ INAF, Istituto di Astrofisica Spaziale e Fisica Cosmica, \\ Via U. La Malfa 153, I-90146 Palermo, Italy \\ 'INAF, Osservatorio Astronomico di Cagliari, \\ Località Poggio dei Pini, strada 54, I-09012 Capoterra, Italy \\ ${ }^{d}$ Department of Astronomy and Astrophysics, Pennsylvania State University, \\ University Park, PA 16802, USA \\ ${ }^{e}$ CRESST/Goddard Space Flight Center, Greenbelt, MD, USA \\ ${ }^{f}$ Universities Space Research Association, Columbia, MD, USA \\ ${ }^{g}$ NASA/Goddard Space Flight Center, Greenbelt, MD 20771, USA \\ ${ }^{h}$ INAF, Osservatorio Astronomico di Brera, \\ via E. Bianchi 46, I-23807 Merate, Italy \\ ${ }^{i}$ Department of Physics \& Astronomy, University of Leicester, LE1 7RH, UK \\ E-mail: sidoli@iasf-milano.inaf.it
}

\begin{abstract}
Supergiant Fast X-ray Transients (SFXTs) are a sub-class of High Mass X-ray Binaries (HMXBs) associated with OB supergiant companions and displaying transient $\mathrm{X}$-ray activity. This behaviour is quite surprising since HMXBs hosting supergiants were known to be persistent sources, until the INTEGRAL discoveries obtained by means of the monitoring of the Galactic plane. We have been performing a monitoring campaign with Swift of four SFXTs with the main aim of characterizing both the long-term behaviour of these transients and the properties during bright outbursts. Here we discuss the properties of the X-ray emission observed outside the outbursts as well as the flares observed from two SFXTs: IGR J17544-2916 and XTE J1739-302. Contrarily to what previously thought, Swift allowed us to discover that SFXTs spend most of the time in accretion at a low level, even outside the bright outbursts, with an accretion luminosity of $10^{33}-10^{34} \mathrm{erg} \mathrm{s}^{-1}$, and that the quiescent level $\sim 10^{32} \mathrm{erg} \mathrm{s}^{-1}$, is a much rarer state.
\end{abstract}

7th INTEGRAL Workshop

September 8-11 2008

Copenhagen, Denmark

${ }^{*}$ Speaker. 


\section{Supergiant Fast $\mathbf{X}$-ray Transients before $S w i f t$}

The Galactic plane monitoring performed with the INTEGRAL satellite led to the discovery of several new sources (Bird et al., 2007). Some of them displayed sporadic, recurrent, bright and short flares, with a typical duration of a few hours and reaching a peak luminosity of $10^{36}$ $10^{37} \mathrm{erg} \mathrm{s}^{-1}$ (Sguera et al, 2005, 2006; Negueruela et al. 2006). Refining the INTEGRAL positions at arcsec level with X-ray follow-up observations, allowed the association with OB supergiant companions (e.g. Halpern et al. 2004; Pellizza et al. 2006; Masetti et al. 2006; Negueruela et al. 2006b; Nespoli et al. 2008).

Other important properties are the spectral similarity with accreting pulsars (hard power law spectra with a high energy cut-off around $15-30 \mathrm{keV}$ ) and the large dynamic range, from a peak luminosity of $10^{36}-10^{37} \mathrm{erg} \mathrm{s}^{-1}$, down to a quiescent emission of $10^{32} \mathrm{erg} \mathrm{s}^{-1}$. The two main characterizing properties (the transient $\mathrm{X}$-ray emission and the association with supergiant companions) indicate that these transients form a new class of High Mass X-ray Binaries, later called Supergiant Fast X-ray Transients (SFXTs; e.g. Negueruela et al. 2006).

The similarities of the SFXTs with the properties of accreting pulsars suggest that the majority of these transients are indeed HMXBs hosting a neutron star, although only in three SFXTs X-ray pulsations have been discovered: IGR J11215-5952 ( $P_{\text {spin }} \sim 186.8 \mathrm{~s}$, Swank et al. 2007); AX J1841.0-0536 ( $P_{\text {spin }} \sim 4.7$ s, Bamba et al. 2001) and IGR J18483-0311 ( $P_{\text {spin }} \sim 21$ s, Sguera et al. 2007).

The confirmed SFXTs are eight (IGR J08408-4503, IGR J11215-5952, IGR J16479-4514, XTE J1739-302, IGR J17544-2619, SAX J1818.6-1703, AX J1841.0-0536 and IGR J184830311), with $\sim 15$ more candidates which showed short transient flaring activity, but with no confirmed association with an OB supergiant companion.

The main mechanisms proposed to explain the short and bright flaring activity from SFXTs deal with the properties of the accretion from the supergiant wind (see Sidoli 2008 for a review), either related with the wind structure (in't Zand 2005; Walter \& Zurita Heras, 2007; Negueruela et al. 2008; Sidoli et al. 2007) or to gated mechanisms which allow accretion onto the neutron star surface only when the centrifugal or the magnetic barriers are open, depending on the values of the neutron star spin and surface magnetic field (e.g. Bozzo et al. 2008 and references therein).

The properties of the SFXTs outbursts, although sporadic and short, have been studied more in depth than the quiescent state. The observations performed outside the bright outbursts have been indeed only a few and short (a few ks long), and caught these sources either in a low level flaring activity (IGR J17544-2619, Gonzalez-Riestra et al. 2004) or in quiescence (with a very soft spectrum, likely thermal, with an X-ray luminosity of $\sim 10^{32} \mathrm{erg} \mathrm{s}^{-1}$ ). Note that this latter quiescent state has been observed only in a couple of SFXTs, IGR J17544-2619 (in't Zand 2005) and IGR J08408-4503 (Leyder et al. 2007).

\section{Swift monitoring of Supergiant Fast X-ray Transients}

Before the Swift campaign (which is still in progress since October 2008) no long-term monitoring of SFXTs have ever been performed to study the status where these transients spend most 
of their life. Nevertheless, it has been assumed by several authors, without observational evidence, that SFXTs spend most of the time in quiescence, when they are not in bright outburst.

The first observations with Swift of a member of this new class of sources have been performed during the 2007 February outburst of IGR J11215-5952 (Romano et al. 2007). This outburst could be completely monitored thanks to its predictability, because IGR J11215-5952 was the first SFXT where periodically recurrent outbursts were discovered (Sidoli et al. 2006). These observations are one of the most complete set of observations of a SFXT in outburst, and clearly demonstrate, for the first time, that the short (a few hours long) flares observed with INTEGRAL (or RXTE, in a few sources), are actually part of a much longer outburst event lasting a few days, implying that the accretion phase lasts longer than what was previously thought (Romano et al. 2007; Sidoli et al. 2007).

The success of this campaign led us to propose with Swift the first wide-band, long-term and deep monitoring campaign of a sample of four SFXTs, with the main aim of (1)-studying the long-term properties of these transients, (2)-performing a truly simultaneous spectroscopy ( $0.3-$ $150 \mathrm{keV}$ ) during outbursts, (3)-studying the outburst recurrence and their durations (see also Romano et al. 2008b, these proceedings). The 4 targets are: XTE J1739-302, IGR J17544-2619, IGR J16479-4514 and AX J1841.0-0536/IGR J18410-0535. The Swift campaign consists of 2-3 observations/week/source (each observation lasts 1-2 ks; see Romano et al. 2008b, these proceedings, for the campaign strategy). Fig. 1 shows the four Swift/XRT light curves (0.2-10 keV) accumulated in the period October 2007-September 2008.

Here we report on the entire Swift monitoring campaign, updated to 2008 September 10. In particular, we focus on the out-of-outburst behaviour (Sidoli et al. 2008a, hereafter Paper I) and on the bright flares observed from two SFXTs of the sample, XTE J1739-302 and IGR J17544-2619 (Sidoli et al. 2008b, hereafter Paper III; Sidoli et al. in preparation). Another outburst caught during this campaign from IGR J16479-4514 was published by Romano et al. (2008a, Paper II).

Preliminary results from the last outbursts from XTE J1739-302 (triggered on 2008 August 13, Romano et al. 2008c) and from IGR J17544-2619 (triggered on 2008 September 4, Romano et al. 2008d) are also discussed here for the first time. A complete analysis will be addressed in Sidoli et al. (in preparation).

\subsection{SFXTs: the long-term X-ray emission outside the bright outbursts}

The SFXTs light curves of Fig. 1 show a clear evidence for highly variable source fluxes even outside the bright outbursts (which were caught in three of the four sources we are monitoring). The light curve variability is on timescales of days, weeks and months, with a dynamic range (outside bright outbursts) of more than one order of magnitude in all four SFXTs. These sources spend most of the time in a frequent low-level flaring activity with an average $2-10 \mathrm{keV}$ luminosity of about $10^{33}-10^{34} \mathrm{erg} \mathrm{s}^{-1}$ (see Paper I).

The average spectra of this out-of-outburst emission are hard (although not as hard as during the bright flares) and can be fitted with an absorbed power law with a photon index in the range 12. The absorbing column density is typically higher than the Galactic value, which can be derived from the optical extinction toward the optical counterparts.

The out-of-outburst emission in IGR J16479-4514 and in AX J1841.0-0536 appears to be modulated with a periodicity in the range 22-25 days, although a full timing analysis will be 
addressed at the end of the campaign. The spectral properties together with the high dynamic range in the flux variability when the sources are not in outburst, demonstrate that SFXTs still accrete matter even outside their bright outbursts, and that the quiescent state (characterized by a very soft spectrum and by a low level of emission at about $10^{32} \mathrm{erg} \mathrm{s}^{-1}$ ) is not the typical long-term state in SFXTs.

\subsection{SFXTs: bright flares from IGR J17544-2619 and XTE J1739-302}

Typically, the SFXTs long-term light curves show a number of bright outbursts, reaching peak luminosities of a few $10^{36} \mathrm{erg} \mathrm{s}^{-1}$, assuming the distances determined by Rahoui et al. (2008). The only source which did not undergo bright flares is AX J1841.0-0536/IGR J18410-0535, which showed a flux variability of more than two orders of magnitude.

During the Swift campaign, three and two outbursts were caught respectively from IGR J175442619 (the first of them triggered BAT, but could not be observed with Swift/XRT because of Sunconstraints) and from XTE J1739-302, at the following dates: on 2007 November 8, 2008 March 31 and 2008 September 4 from IGR J17544-2619, and on 2008 April 8 and 2008 August 13 from XTE J1739-302. Thus, bright flares in this two prototypical SFXTs occur on a timescale of $\sim 4-5$ months (the three outbursts from IGR J17544-2619 were spaced by 144 and 157 days, respectively, while the two outbursts from XTE J1739-302 were spaced by 127 days).

The bright flare from IGR J17544-2619 (on 2008 March 31; Paper III) could be observed simultaneously with XRT (0.2-10 keV) and BAT (15-150 keV). A fit with a power law with a high energy cut-off $\left(e^{\left(E_{\text {cut }}-E\right) / E_{\text {fold }}}\right)$ resulted in the following parameters: $N_{\mathrm{H}}=(1.1 \pm 0.2) \times 10^{22} \mathrm{~cm}^{-2}$, $\Gamma=0.75 \pm 0.11$, cut-off energy $E_{\text {cut }}=18 \pm 2 \mathrm{keV}$ and e-folding energy $E_{\text {fold }}=4 \pm 2 \mathrm{keV}$, reaching a luminosity of $5 \times 10^{37} \mathrm{erg} \mathrm{s}^{-1}(0.5-100 \mathrm{keV}$ at $3.6 \mathrm{kpc})$. Note that the out-of-outburst emission observed with XRT below $10 \mathrm{keV}$ is softer and more absorbed than the emission during this flare.

The other flare observed from IGR J17544-2619 on 2008 September 4 was even brighter (Romano et al. 2008d), and was preceeded by intense activity for a few days as observed with INTEGRAL during the Galactic bulge monitoring programme (Kuulkers et al. 2008; Romano et al. 2008d). The XRT light curve exceeded $20 \mathrm{~s}^{-1}$. This peak emission could be fitted with an absorbed power law with a photon index of $1.3 \pm 0.2$ and an absorbing column density of $1.8_{-0.3}^{+0.4} \times 10^{22} \mathrm{~cm}^{-2}$. The average flux in the $2-10 \mathrm{keV}$ range was $8 \times 10^{-10} \mathrm{erg} \mathrm{cm}^{-2} \mathrm{~s}^{-1}$. The fainter X-ray emission during the flare $\left(2 \times 10^{-10} \mathrm{erg} \mathrm{cm}^{-2} \mathrm{~s}^{-1}\right)$ displayed a similar absorbing column density of $1.4_{-0.5}^{+0.7} \times 10^{22} \mathrm{~cm}^{-2}$ and a photon index $\Gamma=0.8_{-0.3}^{+0.4}$. A more detailed analysis of the properties of this outburst will be performed in a forthcoming paper (Sidoli et al. in preparation).

The first outburst from XTE J1739-302 was caught on 2008 April 8 (Paper III) and was composed by two bright flares separated by about $6000 \mathrm{~s}$. The X-ray emission was significantly more absorbed than in IGR J17544-2619: the broad band (XRT+BAT) spectrum could be well described by an absorbed high energy cut-off power law with the following parameters: $N_{\mathrm{H}}=1.3 \times 10^{23} \mathrm{~cm}^{-2}$, $\Gamma=1.4_{-1.0}^{+0.5}$, cut-off energy $E_{\text {cut }}=6_{-6}^{+7} \mathrm{keV}$ and e-folding energy $E_{\text {fold }}=16_{-8}^{+12} \mathrm{keV}$. The derived X-ray luminosity is $3 \times 10^{37} \mathrm{erg} \mathrm{s}^{-1}(0.5-100 \mathrm{keV})$.

A new outburst was caught from XTE J1739-302 on 2008 August 13 (Romano et al. 2008c). A preliminary spectral analysis of the average broad band spectrum of this bright flare resulted in the following parameters, adopting an absorbed power law with a high energy cut-off: absorbing column density $N_{\mathrm{H}}=(4.0 \pm 0.3) \times 10^{23} \mathrm{~cm}^{-2}, \Gamma=0.7 \pm 0.1, E_{\text {cut }}=4.6 \pm 0.3 \mathrm{keV}$ and $E_{\text {fold }}=9{ }_{-1}^{+2} \mathrm{keV}$. 
The X-ray luminosities during the flare were $2 \times 10^{36} \mathrm{erg} \mathrm{s}^{-1}(0.5-10 \mathrm{keV})$ and $5 \times 10^{36} \mathrm{erg} \mathrm{s}^{-1}$ $(0.5-100 \mathrm{keV})$. Fig. 2 shows the comparison of the spectroscopy in the soft energy range (XRT data) of the out-of-outburst emission with the results from the two flares from XTE J1739-302. A time resolved spectral analysis during the flare will be reported in a forthcoming paper (Sidoli et al., in preparation).

A comparison of the SFXTs light curves (the four SFXTs constantly monitored with Swift, together with other two sources, IGR J11215-5952 and IGR J08408-4503) during their outbursts are reported in Fig. 3. This plot clearly demonstrates that the outbursts from all these transients last much longer than simply a few hours as previously thought. Fig. 3 shows about 8 days of monitoring for each target, and it is clear that the first SFXT, where a day-long outburst event has been observed (IGR J11215-5952, Romano et al. 2007), is not a peculiar case among SFXTs, but a similar behaviour has been observed in the other SFXTs monitored by Swift during the last year (except AX J1841.0-0536, where no outburst have yet been observed).

\section{Conclusions}

The results of the monitoring campaign we have been performing in the last year with Swift of a sample of 4 SFXTs can be summarized as follows:

- the long-term behaviour of the SFXTs outside their outbursts is a low-level accretion phase at a luminosity of $10^{33}-10^{34} \mathrm{erg} \mathrm{s}^{-1}$, with a dynamic range of 1 up to, sometimes, 2 orders of magnitude in flux;

- the broad band X-ray emission during the bright flares can be described well with models commonly adopted for the emission from the accreting X-ray pulsars;

- the SFXTs spectra during flares show high energy cut-offs compatible with a neutron star magnetic field of about $10^{12} \mathrm{G}$, although no cyclotron lines have been detected yet;

- the duration of the outbursts from different SFXTs observed with Swift are longer than a few hours.

\section{Acknowledgments}

We thank the Swift team duty scientists and science planners P.J. Brown, M. Chester, E.A. Hoversten, S. Hunsberger, C. Pagani, J. Racusin, and M.C. Stroh for their dedication and willingness to accomodate our sudden requests in response to outbursts during this long monitoring effort. We also thank the remainder of the Swift XRT and BAT teams, J.A. Nousek and S. Barthelmy in particular, for their invaluable help and support with the planning and execution of the observing strategy. This work was supported in Italy by contracts ASI I/023/05/0, I/088/06/0, and I/008/07/0, at PSU by NASA contract NAS5-00136. H.A.K. was supported by the Swift project. P.R. thanks INAF-IASF Milano and L.S. INAF-IASF Palermo, for their kind hospitality. Italian researchers acknowledge the support of Nature $(455,835-836)$ and thank the Editors for increasing the international awareness of the current critical situation of the Italian Research. 


\section{References}

[1] Bamba, A., Yokogawa, J., Ueno, M., et al., 2001, PASJ, 53, 1179

[2] Bird, A., Malizia, A., Bazzano, A., 2007, ApJS, 170, 175

[3] Bozzo, E., Falanga, M., Stella, L., 2008, ApJ, 683, 1031

[4] González-Riestra, R., Oosterbroek, T., Kuulkers, E., et al., 2004, A\&A, 420, 589

[5] Halpern, J.P., Gotthelf, E.V., Helfand, D.J., et al. 2004, The Astronomer's Telegram, 289

[6] in’t Zand, J.J.M., 2005, A\&A, 441, L1

[7] Kuulkers, E., Risquez Oneca, D., Brandt, S., et al., 2008, The Astronomer's Telegram, 1266

[8] Leyder, J.-C., Walter, R., Lazos, M., et al., 2007, A\&A, 465, L35

[9] Negueruela, I., Smith, D.M., Reig, P., et al. 2006a, in ESA Special Publication, ed. A.Wilson, Vol. 604, 165-170

[10] Negueruela, I., Torrejon, J.M., Reig, P., et al., 2008, AIPC, 1010, 252

[11] Masetti, N., Morelli, L., Palazzi, E., et al. 2006, $A \& A$, 459, 21

[12] Nespoli, E., Fabregat J., Mennickent, R.E., 2008, A\&A, 486, 911

[13] Pellizza, L.J., Chaty, S., Negueruela, I., 2006, A\&A, 455, 653

[14] Rahoui, F., Chaty, S., Lagage, P.-O., et al., 2008, A\&A, 484, 801

[15] Romano, P., Sidoli, L., Mangano, V. et al., 2007a, $A \& A, 469$, L5

[16] Romano, P., Mangano, V., Mereghetti, S., et al., 2007b, The Astronomer's Telegram, 1151

[17] Romano, P., Sidoli, L., Mangano, V., et al., 2008a, ApJ, 680, L137 (Paper II)

[18] Romano, P., Sidoli, L., Mangano, V., et al., 2008b, these proceedings,'PoS(Integral08)088.'

[19] Romano, P., Guidorzi, C., Sidoli, L., et al., 2008c, The Astronomer's Telegram, 1659

[20] Romano, P., Cusumano, G., Sidoli, L., et al., 2008d, The Astronomer's Telegram, 1697

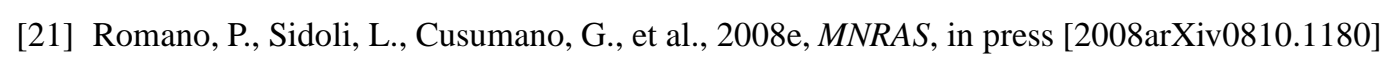

[22] Sguera, V., Barlow, E.J., Bird, A.J., et al. 2005, $A \& A, \mathbf{4 4 4}, 221$

[23] Sguera, V., Bazzano, A., Bird, A. J., et al. 2006, ApJ, 646, 452

[24] Sidoli, L., Paizis, A., \& Mereghetti, S., 2006, A\&A, 450, L9

[25] Sidoli, L., Romano, P., Mereghetti, S., et al., 2007, A\&A, 476, 1307

[26] Sidoli, L., Romano, P., Mangano, V., et al., 2008a, ApJ in pressi[arXiv0805.1808, Paper I];

[27] Sidoli, L., Romano, P., Mangano, V., et al., 2008b, ApJ in pressi[arXiv0808.3085, Paper III]';

[28] Sidoli, L., 2008, Proc. of the COSPAR Assembly 2008, 37, 2892,

[29] Sguera, V., Hill, A. B., Bird, A. J., et al., 2007, $A \& A$, 467, 249

[30] Swank, J.H., Smith, D.M., Markwardt, C.B., 2007, The Astronomer's Telegram, 999

[31] Walter, R., \& Zurita Heras, J., 2007, A\&A, 476, 335 

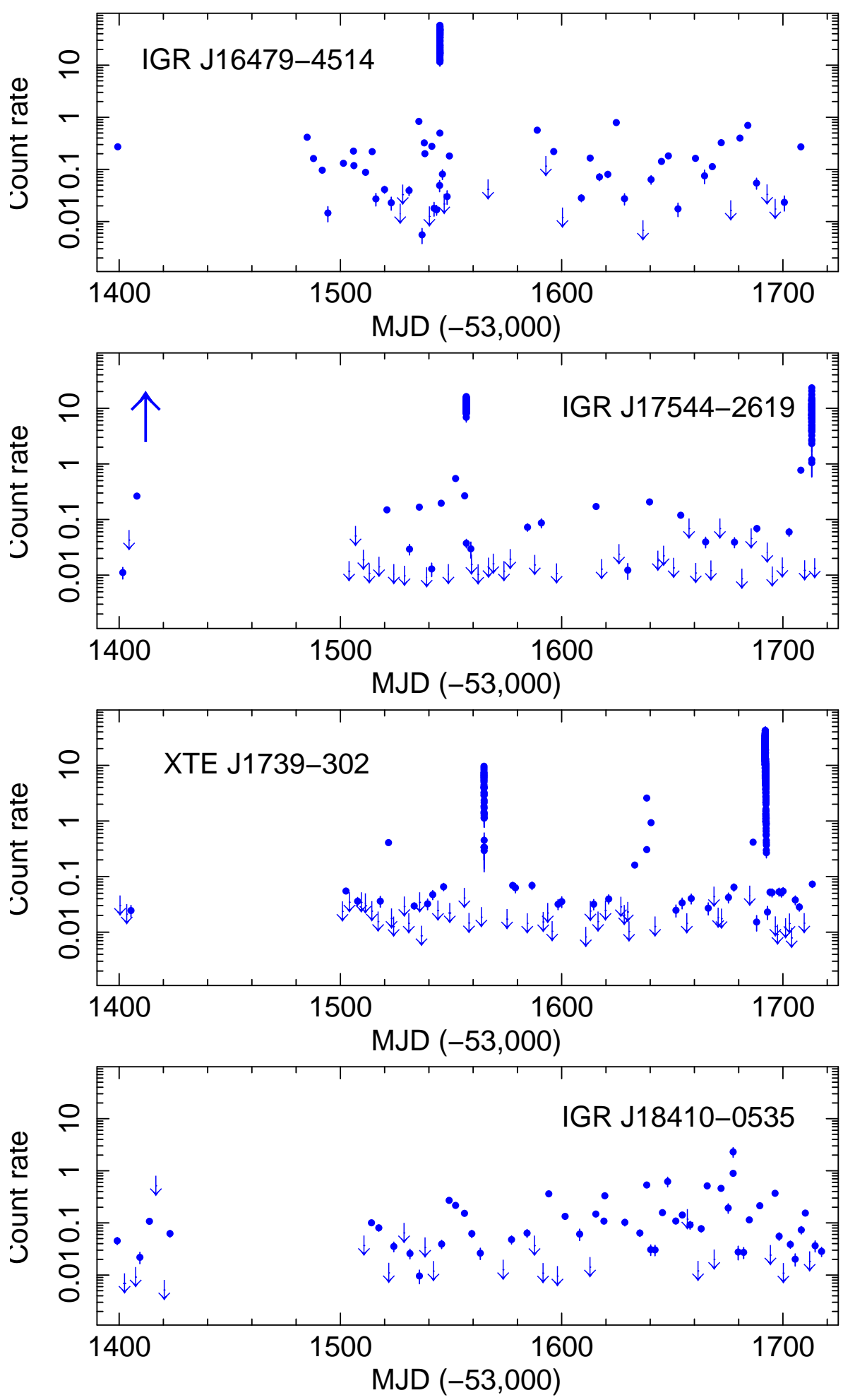

Figure 1: Light curves of the 4 SFXTs monitored with Swift/XRT $(0.2-10 \mathrm{keV})$, from 2007 October to 2008 September 10. The upward pointing arrow in the IGR J17544-2619 light curve marks an outburst which triggered the BAT Monitor on MJD 54412 (200711-08) but could not be observed with XRT because the source was Sun-constrained. The downward-pointing arrows are 3- $\sigma$ upper limits. The gap in the observations between about December 2007 and January 2008 is because the sources were Sun-costrained. 


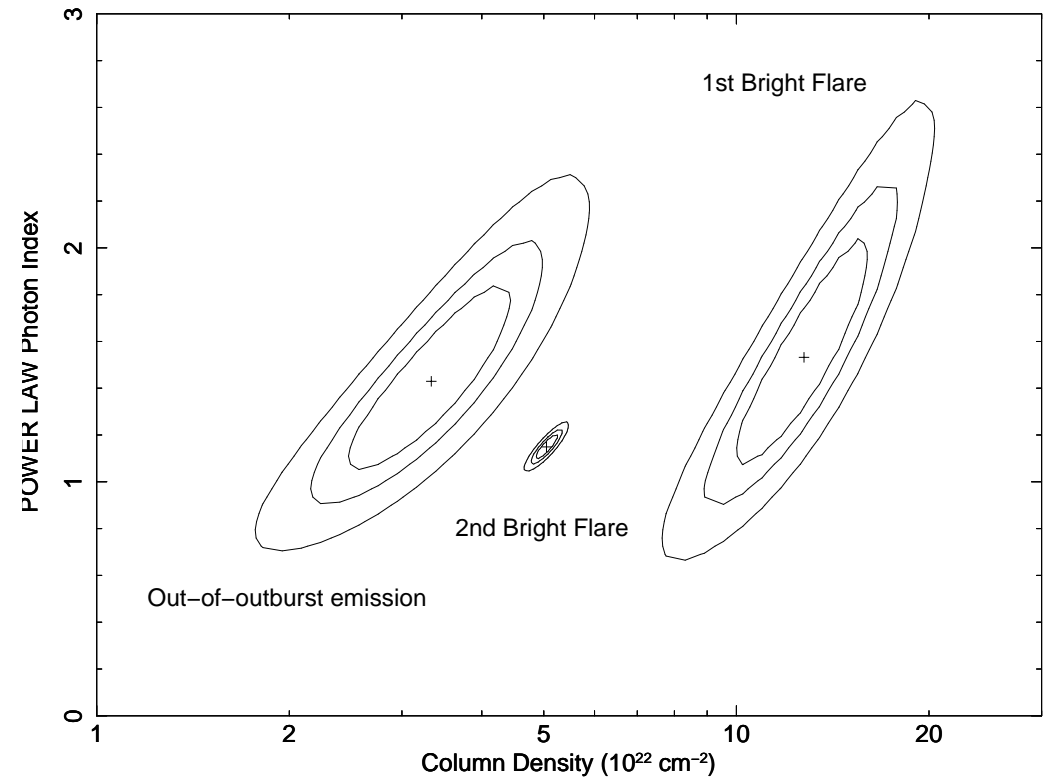

Figure 2: Comparison of the spectral paramenters (absorbed single power law model) derived for XTE J1739-302 during the two bright flares discussed here, and the total spectrum of the out-of-outburst emission reported in Paper I. $68 \%, 90 \%$ and $99 \%$ confidence level contours are shown. 


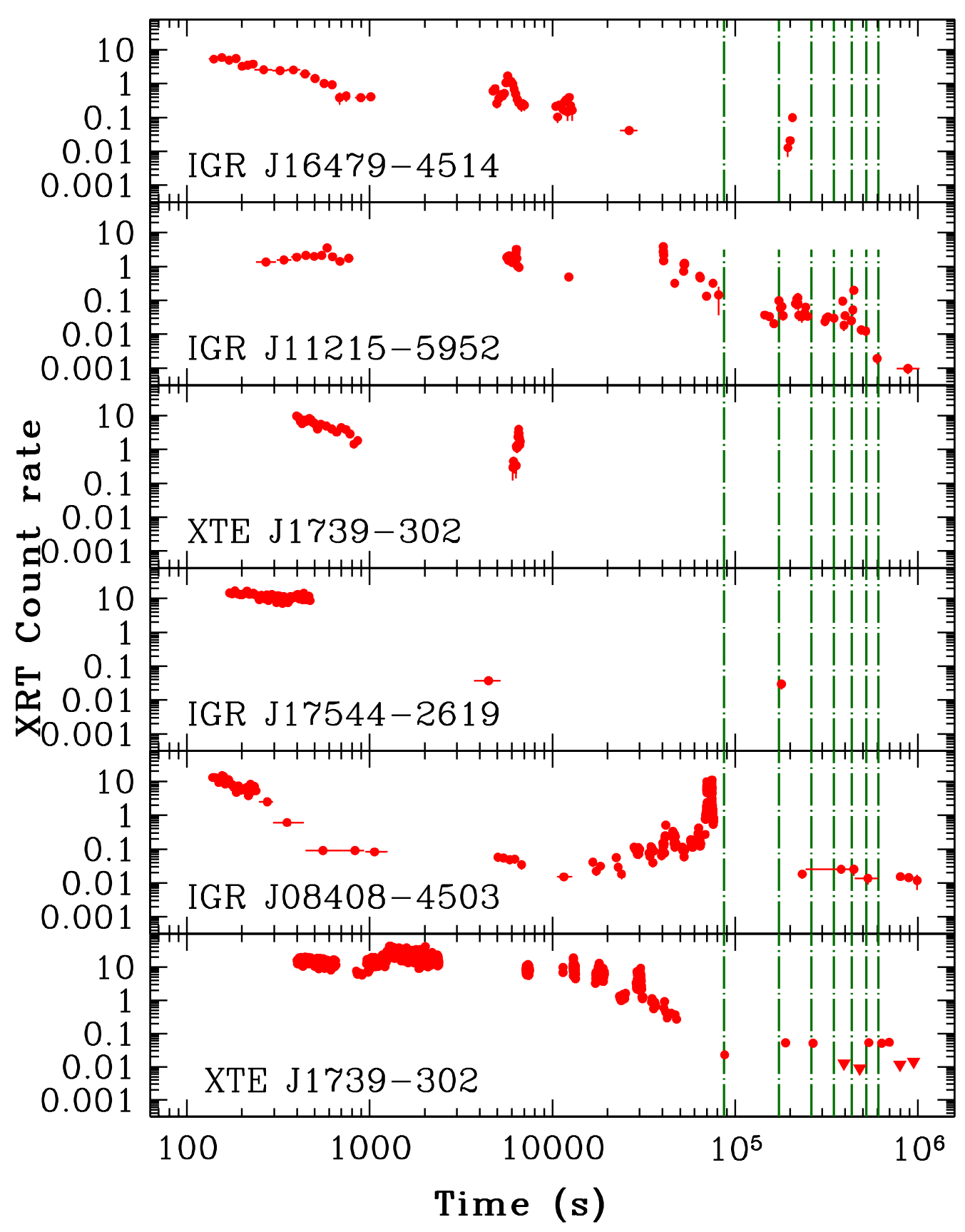

Figure 3: Light curves of the outbursts of SFXTs followed by Swift/XRT referred to their respective triggers. We show the 2005 outburst of IGR J16479-4514 (Paper I), which is more complete than the one observed in 2008 (Paper II). The IGR J11215-5952 light curve has an arbitrary start time, since the source did not trigger the BAT (the observations were obtained as a ToO; Romano et al. 2007). The third and the last panels report the two flares from XTE J1739-302 observed on 2008 April 8 and on 2008 August 13, respectively. The forth panel shows the outburst from IGR J17544-2916 occurred on 2008 March 31 (Paper III). The fifth panel reports on a multiple flaring activity reported from another SFXT, not part of this campaign, IGR J08408-4503, and occurred on 2008 July 5 (Romano et al., 2008e). Note that where no data are plotted, no data were collected. Vertical dashed lines mark time intervals equal to 1 day. 